\title{
Protein Tyrosine Phosphatase H1 (PTPH1) Inhibits LPS-induced TNF- $\alpha$ Production
}

\author{
Huiyun Seo, In-Seon Lee, and Sayeon Cho* \\ College of Pharmacy, Chung-Ang University, Seoul 156-756, Korea. E-mail: sycho@cau.ac.kr \\ Received October 4, 2010, Accepted October 23, 2010
}

Key Words: Lipopolysaccharide, TNF- $\alpha$, Protein tyrosine phosphatase H1, Mitogen-activated protein kinase

Protein-tyrosine-phosphatases (PTPs) play critical roles in signal transduction pathways that regulate fundamental cellular processes such as differentiation, cell proliferation, apoptosis, cellular adhesion, cell cycle progression, and immunological signaling. ${ }^{1-3}$ Protein phosphorylation is regulated by the opposing actions of PTPs and protein tyrosine kinases (PTKs) and provides an important means of regulating protein function. The regulated activity and expression of PTPs in different cells control phosphorylation intensity and duration to determine the type of physiological response. PTP/PTK action on immune response can be either positive or negative, promoting or inhibiting the immune system, and PTP-deficient mice have been used to identify individual PTPs as key regulators of immune responses. In lipopolysaccharide (LPS)-stimulated RAW264.7 cells, mitogen-activated protein kinase (MAPK) phosphatase-1 (MKP-1, also known as dual specificity phosphatase 1 (DUSP1)) induction is associated with inactivation of c-Jun N-terminal kinase (JNK) and p38. ${ }^{4}$ Also, Dusp $1^{-/}$macrophages had elevated p38 and JNK activities but no change in extracellular signal-regulated kinase (ERK) activity. ${ }^{5,6}$ Protein tyrosine phosphatase H1 (PTPH1) (also known as protein tyrosine phosphatase nonreceptor type 3 (PTPN3)) contains two major domains, a PDZ and band 4.1 domain. $^{7-9}$ Band 4.1 domains are responsible for targeting proteins to the cytoskeleton-membrane interface. ${ }^{10}$ PDZ domains have been implicated in mediating protein-protein interactions, recognizing C-terminal valine residues, and binding to other PDZ domains. ${ }^{11}$ Expression of active PTPH1 in Jurkat T cells reduces phosphorylation of MEK, ERK, JNK and AP-1 indirectly, leading to a decreased IL-2 gene activation. ${ }^{12}$ TNF- $\alpha$ is produced by a variety of immune cells including B cells, T cells, NK cells, and macrophages. ${ }^{13}$ TNF- $\alpha$ expression is regulated by activation of kinase pathways (including ERK, JNK, p38 MAPK, PKC, and Akt) and leads to activation of transcription factors such as AP-1 and NF- $\kappa \mathrm{B} .{ }^{14}$

In the present study, we investigated the effect of PTPH1 on TNF- $\alpha$ induction in an effort to identify more PTPs that regulate TNF- $\alpha$ expression. To examine the effect of PTPH1 on the production of proinflammatory cytokines, a mammalian vector bearing FLAG-tagged PTPH1 was transfected into RAW264.7 cells by FuGENE HD transfection reagent. The effect of increased PTPH1 expression on the production of TNF- $\alpha$ was examined by ELISA. Cells transfected with FLAG-PTPH1 plasmid produce less TNF- $\alpha$ after LPS treatment than cells transfected with empty plasmid, suggesting that PTPH1 might be involved in the regulation of TNF- $\alpha$ expression induced by LPS (Fig. 1). TNF- $\alpha$ production was further reduced when more FLAG-PTPH1 plasmid was transfected. Therefore, our results

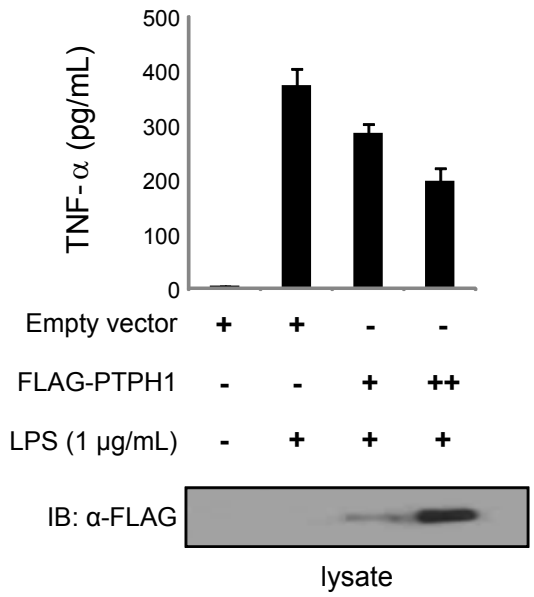

Figure 1. Inhibition of LPS-stimulated TNF- $\alpha$ production by PTPH1 in macrophages. RAW264.7 cells were transfected with either the empty vector or the construct bearing FLAG-PTPH1. After $16 \mathrm{~h}$ stimulation with LPS, supernatants were analyzed for TNF- $\alpha$ production using an ELISA assay, as described in the Experimental Section. Cell lysates were subjected to immunoblotting using an anti-FLAG antibody for detection of PTPH1. The results presented are representative of three independent experiments. Error bars indicate \pm SEM.

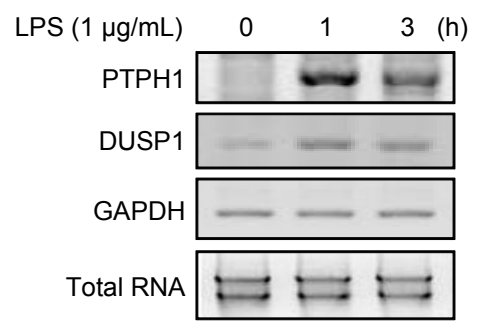

Figure 2. Induction of PTPH1 in RAW264.7 cells after treatment with LPS. Expression levels of PTPH1 in RAW264.7 cells were determined at the indicated times after treatment of LPS $(1 \mu \mathrm{g} / \mathrm{mL})$ by RT-PCR. GAPDH transcripts were amplified using specific primers (forward 5'-ACCACCATGGAGAAGGC-3'; reverse 5'-CTCAGTGTAGCCC AGG ATGC-3') as a control for RNA integrity. DUSP1 transcripts were amplified and used as a positive control for LPS treatment.

indicate that PTPH1 inhibits TNF- $\alpha$ production in LPS-treated macrophages.

Next, we tested if PTPH1 gene expression is regulated by LPS in RAW264.7 cells. PTPH1 mRNA expression levels were determined by RT-PCR using mRNAs obtained from RAW264.7 cells stimulated with LPS or untreated. In LPS-stimulated RAW 264.7 cells, DUSP1 expression, which was used as a control, 


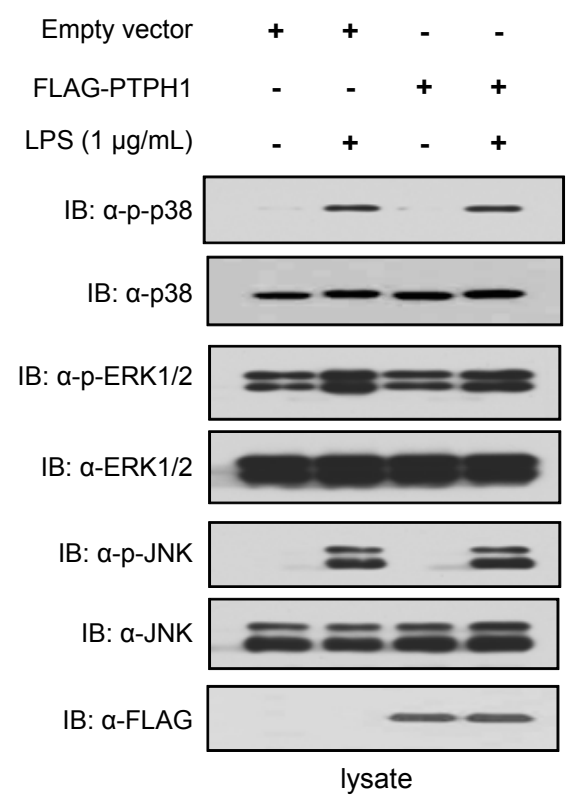

Figure 3. No effect of PTPH1 on LPS-mediated MAPK signal transduction. PTPH1 expression plasmid was transfected into RAW264.7 cells and then cells were stimulated with LPS $(1 \mu \mathrm{g} / \mathrm{mL})$ for $1 \mathrm{~h}$. Immunoblottings were performed for total and phosphorylated proteins as indicated. Three independent experiments were performed with similar results.

reached its maximal level at $1 \mathrm{~h}$, and then decreased slightly as shown Fig. 2. Likewise, PTPH1 mRNA levels also increased dramatically in RAW 264.7 cells in response to LPS (Fig. 2). These results indicate that PTPH1 expression is regulated by LPS.

Since MAPKs play major roles in TNF- $\alpha$ production in RAW 264.7 cells, we examined the relationship between PTPH1 expression and MAPK inactivation. RAW264.7 cells were transiently transfected with FLAG-tagged PTPH1 expression plasmids or empty plasmids. We then assessed the endogenous levels of phospho-JNK, phospho-p38, and phospho-ERK1/2 using immunoblotting analysis. LPS induced phosphorylation of p38, JNK, and ERK. However, overexpression of PTPH1 showed no change in phosphorylation of MAPKs in RAW264.7 cells when compared with cells transfected with empty plasmid (Fig. 3). Taken together, these results suggest that PTPH1 had no effect on MAPK pathway and might be involved in other signaling pathways such as NF- $\kappa \mathrm{B}$. Young et al reported that PTPH1 negatively regulates NF- $\mathrm{B}$ signaling. ${ }^{15}$

In conclusion, the results of this study suggest the role of PTPH1 in the inflammatory response of macrophages, particularly its influence on the production of proinflammatory cytokines. PTPH1 was potently induced in response to LPS stimulation in RAW264.7 cells and might be involved in the negative feedback regulation of TNF- $\alpha$ production.

\section{Experimental Section}

Cell culture and transfection. The murine macrophage RAW 264.7 cells were maintained at $37{ }^{\circ} \mathrm{C}$ in Dulbecco's modified Eagle's medium (DMEM, Invitrogen, Carlsbad California) su- pplemented with $10 \%$ fetal bovine serum (FBS, Invitrogen) and penicillin/streptomycin in the presence of $5 \% \mathrm{CO}_{2}$. For transient transfection, $1.4 \times 10^{6}$ cells were plated in each $60-\mathrm{mm}$ cell culture plate, grown overnight, and transfected with DNA using FuGENE HD transfection reagent (Roche, Basel, Switzerland).

Plasmid constructs. The N-terminal FLAG-tagged PTPH1 for expression in mammalian cells was constructed by polymerase chain reaction, followed by cloning into the pcDNA3.1/ Zeo plasmid.

Reagents and antibodies. Polyclonal anti-JNK, anti-phospho-JNK (Thr-183/Tyr-185), anti-p38, anti-phospho-p38 (Thr180/Tyr-182), anti-ERK1/2, and anti-phospho-ERK1/2 (Thr202/Tyr-204) antibodies were from Cell Signaling Technology (Danvers, MA). Anti-FLAG M2 antibody and LPS were from Sigma-Aldrich.

TNF- $\alpha$ ELISA. TNF- $\alpha$ protein concentrations were determined by standard sandwich ELISA using antibodies and standards obtained from BD Biosciences (San Diego, CA) and used according to manufacturer's instructions. Assays were performed on neat and diluted samples in duplicate on 96-well plates. Absorbance was measured by a microplate reader at $450 \mathrm{~nm}$ and concentrations were determined by comparison to a standard curve. All transfections were repeated at least three times.

Reverse transcription-polymerase chain reaction (RT-PCR). Total RNAs were prepared from cells by Trizol (Roche) and reverse transcription was performed by using M-MLV (Invitrogen). PCR for mouse PTPH1 was carried out using the following primers: forward 5'-ATACATCATCCAGGCTATC-3' and reverse 5'-GTAATTCAGCATGTTGAAG-3'.

Immunoblotting analysis. After RAW264.7 cells were transiently transfected with FLAG-tagged PTPH1 expression plasmids or empty plasmids for $48 \mathrm{~h}$, cells were washed twice with phosphate buffered saline (PBS) buffer and lysed in PTP lysis buffer $(0.5 \%$ NP-40, $0.5 \%$ Triton X-100, $150 \mathrm{mM} \mathrm{NaCl}, 20 \mathrm{mM}$ Tris- $\mathrm{HCl}$ (pH 8.0), 1 mM EDTA, 1\% glycerol, 1 mM phenylmethylsulfonyl fluoride, and $1 \mu \mathrm{g} / \mathrm{ml}$ Aprotinin) for $30 \mathrm{~min}$ at $4{ }^{\circ} \mathrm{C}$. Cleared cell lysates from centrifugation were resuspended with protein sample buffer, boiled at $100{ }^{\circ} \mathrm{C}$ for $5 \mathrm{~min}$, subjected to SDS-PAGE, and subsequently transferred onto nitrocellulose membrane. Immunoblotting analysis was carried out as previously described. ${ }^{16}$

Acknowledgments. This work was supported by a grant of the Korea Health 21 R\&D Project, Ministry of Health \& Welfare, Republic of Korea (A010385-1033-0000400) and by the Korea Research Foundation Grant funded by the Korean Government (MOEHRD, Basic Research Promotion Fund) (KRF2008-331-E00089).

\section{References}

1. Hunter, T. Cell 2000, 100, 113 .

2. Tonks, N. K.; Neel, B. G. Cell 1996, 87, 365.

3. Denu, J. M.; Stuckey, J. A.; Saper, M. A.; Dixon, J. E. Cell 1996, $87,361$.

4. Chen, P.; Li, J.; Barnes, J.; Kokkonen, G. C.; Lee, J. C.; Liu, Y. J. Immunol. 2002, 169, 6408.

5. Franklin, C. C.; Kraft, A. S. J. Biol. Chem. 1997, 272, 16917. 
6. Dorfman, K.; Carrasco, D.; Gruda, M.; Ryan, C.; Lira, S. A.; Bravo, R. Oncogene 1996, 13, 925.

7. Yang, Q.; Tonks, N. K. Proc. Natl. Acad. Sci. USA 1991, 88, 5949.

8. Gu, M. X.; York, J. D.; Warshawsky, I.; Majerus, P. W. Proc. Natl. Acad. Sci. USA 1991, 88, 5867.

9. Zhang, S. H.; Kobayashi, R.; Graves, P. R.; Piwnica-Worms, H.; Tonks, N. K. J. Biol. Chem. 1997, 272, 27281.

10. Arpin, M.; Algrain, M.; Louvard, D. Curr. Opin. Cell. Biol. 1994, 6,136 .
11. Ponting, C. P.; Phillips, C.; Davies, K. E.; Blake, D. J. Bioessays 1997, 19, 469 .

12. Han, S.; Williams, S.; Mustelin, T. Eur. J. Immunol. 2000, 30, 1318.

13. Aggarwal, B. B. Nat. Rev. Immunol. 2003, 3, 745.

14. Hehlgans, T.; Pfeffer, K. Immunology 2005, 115, 1.

15. Young, J. A.; Becker, A. M.; Medeiros, J. J.; Shapiro, V. S.; Wang, A.; Farrar, J. D.; Quill, T. A.; Hooft van Huijsduijnen, R.; van Oers, N. S. Mol. Immunol. 2008, 45, 3756.

16. Song, M.; Cho, S. Bull. Korean Chem. Soc. 2009, 30, 1190. 